\title{
Instrumental perforation of the oesophagus into the pericardial cavity
}

\author{
U R NAIR
}

From the Regional Cardiothoracic Centre, Wythenshawe Hospital, Manchester

Instrumental perforation of the oesophagus is a rare but well known complication of endoscopy, which is more common with the rigid instrument. ${ }^{1}$ Associated perforation of the pericardial sac is an even rarer and potentially fatal condition. We report in this paper the successful management of such a case.

\section{Case report}

A 66 year old man with no previous oesophageal symptoms developed acute dysphagia and vomiting shortly after a large meal. About 16 hours later a large piece of meat was removed from the oesophagus at the $30 \mathrm{~cm}$ level under general anaesthesia through a rigid oesophagoscope. The surgeon who performed the endoscopy explored the distal oesophagus for strictures and noticed that the oesophagoscope would not pass beyond $40 \mathrm{~cm}$. A few hours later the patient developed moderately intense chest pain and surgical emphysema of the lower neck. He was given penicillin and gentamicin intravenously and transferred to the Regional Cardiothoracic Centre for further management.

The patient had a heart rate of 105 beats/min and a

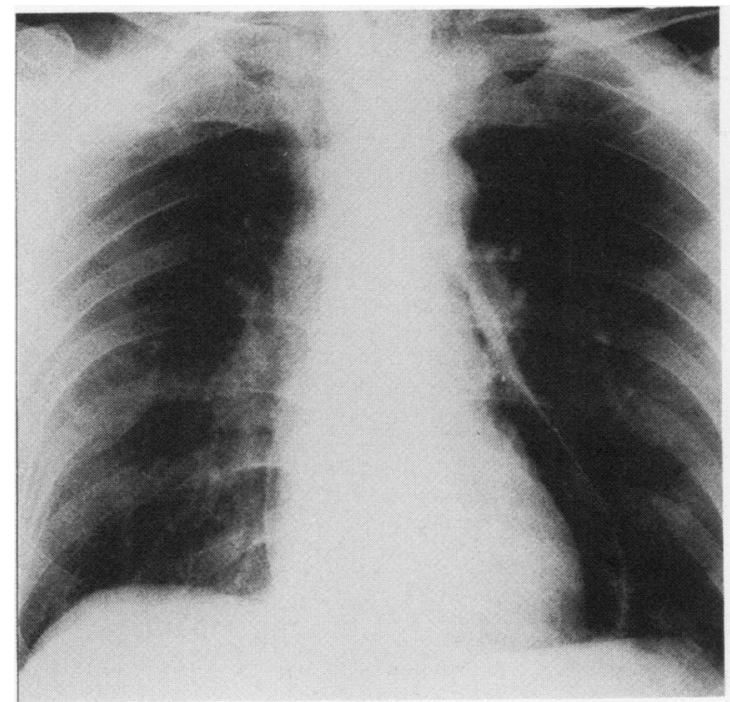

Fig 1 Chest radiograph showing pneumopericardium and pneumomediastinum.

Address for reprint requests: Mr UR Nair FRCS, Regional Cardiothoracic Centre, Wythenshawe Hospital, Manchester M23 9LT. temperature of $37.8^{\circ} \mathrm{C}$ on admission. The chest radiograph showed pneumopericardium and free gas in the mediastinum; both lungs were fully expanded (fig 1). A gastrograffin swallow demonstrated free flow of contrast into the pericardial sac through a perforation at the level of the aortic arch (fig 2). There was no flow of contrast medium into the lower oesophagus. The patient developed acute tamponade soon after this investigation, and was transferred to theatre for repair of the perforation. He was anaesthetised after insertion of a radial arterial monitoring line. A preliminary oesophagoscopy confirmed the presence of a tear at $24 \mathrm{~cm}$. A small nasogastric tube was passed through the perforation for identification at operation. The patient's arterial pressures improved after removal of about $150 \mathrm{ml}$ of dilute contrast medium through the tube. The left chest was explored through the fifth intercostal space, and the nasogastric tube identified in the pericardial sac. The site of perforation was easily located by following the tube upwards. The oesophagus had a $2 \mathrm{~cm}$ tear at the level of the aortic arch and the pericardium was found to be torn between the left atrial appendage and the left pulmonary

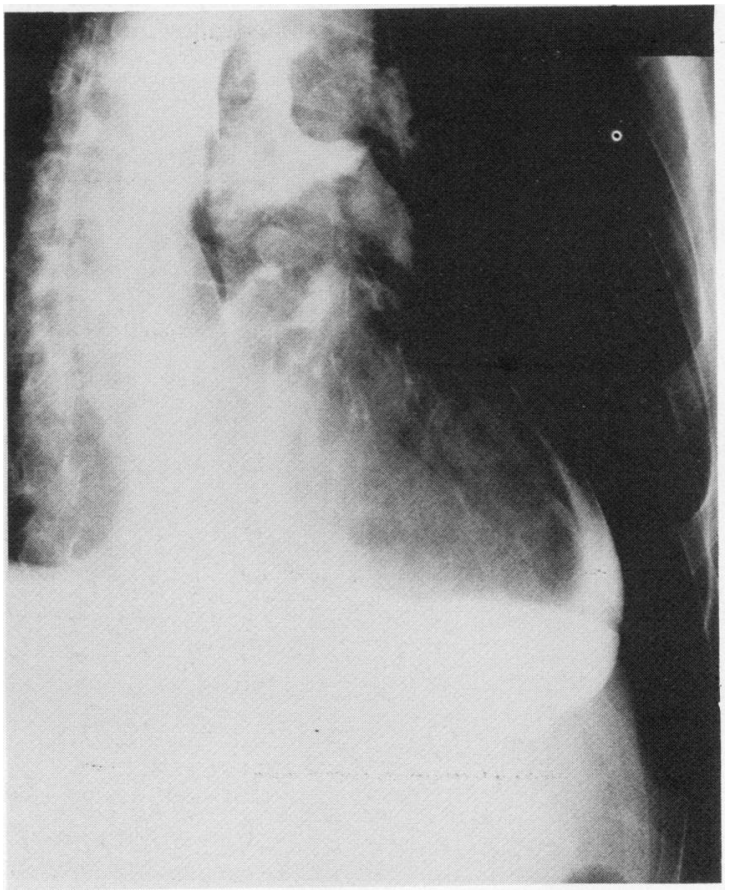

Fig 2 Gastrograffin swallow showing contrast medium in the pericardial cavity. 
artery. There was dissection and inflammation of the tissues under the aortic arch between these two points. The oesophageal tear was exposed by mobilising the aortic arch medially, and was repaired in two layers with interrupted (00) chromic catgut sutures. There was severe inflammation of the pericardial tissues, which were washed clean with an aqueous iodine solution, after which the sac was closed loosely, adequate drainage being left in the lowest part. The lower oesophagus was explored and was found to be normal. The chest wall was then closed in layers over two pleural drains.

The patient recovered uneventfully and was given restricted oral fluids on the fifth day, and free fluids on the seventh day after the operation. He was discharged home on the 10th day in excellent condition.

\section{Discussion}

Instrumental perforation of the oesophagus is seen more commonly in the lower part. $^{2}$ The associated pneumomediastinum is a common feature of intrathoracic perforations. ${ }^{3}$ Subsequent mediastinitis increases the morbidity and risk of death in these patients. Early surgical intervention is advised by many authors, ${ }^{1-5}$ even though a smaller number have been treated conservatively. ${ }^{6}$ Better healing is observed when the distal oesophagus is normal. We have not come across any reported pericardial perforation. The chest radiographic appearances of pneumopericardium should arouse suspicion of this condition, and subsequent investigations with contrast medium are best done after early surgical intervention has been arranged. Our patient became cyanosed and developed cardiac tamponade soon after the gastrograffin swallow, and appre- ciable improvement in arterial pressures was noticed aft the removal of pericardial fluid at oesophagoscopy.

We recommend a preliminary oesophagoscopy in cases of perforation, as the nasogastric tube passed through the perforation was immensely helpful in identifying the exact sef of injury with minimal dissection. The surgical approag should be determined by the direction of the leak, and not the level alone. Repair of the oesophagus through a loft thoracotomy is not difficult, even under the aortic arch, onfe the aortic arch has been mobilised. Pericardial tear predisposes to a higher risk of infection, which could te reduced by adequate cleaning and drainage.

Oesophageal tear extending into the pericardial sac isfa potentially fatal injury, and this case report furtherer emphasises the delicacy with which oesophagoscopy shoifd be performed, especially in a patient under general anaesthesia.

\section{References}

1 Triggiani E, Belsey R. Oesophageal trauma: incidence, diagnos] and management. Thorax 1977;32:241-9.

2 Banks JG, Bancewicz J. Perforation of the oesophagus experience in a general hospital. Br J Surg 1981;68:580-4.

3 Sandrasagara FA, English TAH, Milstein BB. The manageme®n and prognosis of oesophageal perforation. Br $J$ Surg 1978;65:629-32.

4 Wichern WA jun. Perforation of the oesophagus. Am J S S $g$ 1970;119:534-6.

5 Sawyers JL, Lane CE, Foster JH, Daniel RA. Esophageal per foration. Ann Thorac Surg 1975;19:233-8.

6 Wesdorp ICE, Bartelsman JFWM, Huibregtse K, Den Har® Jager FCA, Tytgat GN. Treatment of instrumental oespphageal perforation. Gut 1984;25:398-404. 